\title{
Genome-wide tracts of homozygosity and exome analyses reveal repetitive elements with Barrets esophagus/esophageal adenocarcinoma risk
}

\author{
Visanu Wanchai ${ }^{1}$, Jing Jin ${ }^{2}$, Emine Bircan ${ }^{2}$, Charis Eng ${ }^{4}$ and Mohammed Orloff ${ }^{2,3^{*}}$
}

From The 15th Annual MCBIOS Conference

Starkville, MS, USA. March 29 - 312018

\begin{abstract}
Background: Barrett's esophagus (BE) is most commonly seen as the condition in which the normal squamous epithelium lining of the esophagus is replaced by goblet cells. Many studies show that BE is a predisposing factor for the development of esophageal adenocarcinoma (EAC), a particularly lethal cancer. The use of single nucleotide polymorphisms (SNPs) to map BE/EAC genes has previously provided insufficient genetic information to fully characterize the heterogeneous nature of the disease. We therefore hypothesize that rigorous interrogation of other types of genomic changes, e.g. tracts of homozygosity $(\mathrm{TOH})$, repetitive elements, and insertion/deletions, may provide a comprehensive understanding of the development of BE/EAC.

Results: First, we used a case-control framework to identify TOHs by using SNPs and tested for association with BE/ EAC. Second, we used a case only approach on a validation series of eight samples subjected to exome sequencing to identify repeat elements and insertion/deletions. Third, insertion/deletions and repeat elements identified in the exomes were then mapped onto genes in the significant $\mathrm{TOH}$ regions. Overall, $24 \mathrm{TOH}$ regions were significantly differentially represented among cases, as compared to controls (adjusted- $P=0.002-0.039$ ). Interestingly, four BE/ EAC-associated genes within the $\mathrm{TOH}$ regions consistently showed insertions and deletions that overlapped across eight exomes. Predictive functional analysis identified NOTCH, WNT, and G-protein inflammation pathways that affect BE and EAC.
\end{abstract}

Conclusions: The integration of common TOHs (CTOHs) with repetitive elements, insertions, and deletions within exomes can help functionally prioritize factors contributing to low to moderate penetrance predisposition to BE/EAC.

Keywords: Barrett's esophagus, Esophageal adenocarcinoma, Tracts of homozygosity, Exome, Omics

\section{Background}

Barrett's esophagus, the only known precancerous lesions for esophageal adenocarcinoma (EAC), is characterized as an abnormal replacement of the stratified squamous epithelium in the lower portion of the

\footnotetext{
* Correspondence: msorloff@uams.edu

${ }^{2}$ The Department of Epidemiology, University of Arkansas for Medical Sciences, Little Rock, AR 72205, USA

${ }^{3}$ Winthrop P. Rockefeller Cancer Institute, University of Arkansas for Medical Sciences, Little Rock, AR 72205, USA

Full list of author information is available at the end of the article
}

esophagus with metaplastic, columnar epithelium called goblet cells that can secrete gel-forming mucins [1]. Esophageal cancer is more common among men than women [2]. According to the American Cancer Society's estimate for 2018 [2], the lifetime risk of developing esophageal cancer in the United States is about 1 in 132 for men and about 1 in 455 for women.

Of the two cancer types, EAC and squamous cell carcinoma (SCC), EAC is more prevalent in the United States overall [2], while SCC is observed more often in African Americans [3]. Possible risk factors, other than 
race, have been identified by previous studies; these include aging, male gender, gastroesophageal reflux disease, tobacco use, alcohol use, diet, and human papillomavirus (HPV) [2, 4].

Somatic mutations in EAC have been studied and some of the common mutations have been identified in TP53, CDKN2Ai, BRAF, CTNNB1, EGFR, KRAS, PTEN and $C D H 1$ [2]. Although most cases of BE and EAC are believed to be sporadic, heritable etiologies have been reported as well $[5,6]$. In addition to shared environmental factors, reports on BE and EAC have shown a frequent autosomal dominant mode of inheritance with incomplete penetrance and autosomal recessive inheritance, which are rare [7].

Historically, several types of genetic markers, including SNPs, have been used to map genes to BE/EAC [8]. Polymorphic tracts of nucleotide sequences, such as those of repeat nucleotides of variable lengths $[9,10]$ and tracts of homozygosity (TOHs) [11-15], seem to occur frequently in the human genome. Whereas the importance of SNPs and their associations with disease risk are well established [16, 17], there is an increasing appreciation for the potential role of nucleotide repeats $[9,18]$ and TOHs $[11,14,15,19,20]$ in the risk of developing disease. Nucleotide repeats and TOHs are common in the genome; however, their associations with risk of developing common diseases are understudied [21].

Approximately half of the human genome is composed of highly repeated DNA sequences [22]. Some of these nucleotide repeats (e.g., satellite repeats) have been shown to transcribe into noncoding RNAs, which have been linked to gene silencing and maintenance of chromosomal integrity [23]. The processes that involve inversions and deletions in the genome yield another family of repeats called inverted repeats (or IRs), which are a single stranded sequence of nucleotides that are followed downstream by their reverse complement [24]. If selection pressure favors minimizing inversions, then more direct repeats are expected, relative to IRs [23, 2528]. However, inversions are required to create IRs from direct repeats, if these repeats originate mainly from close direct repeats [29]. The sequences of these IRs have been found to locate near endogenous chromosomal instability and breakage hotspots [21], but the mutagenic potential of IRs has not been well characterized. To the best of our knowledge, the role of repeat sequences in $\mathrm{BE} / \mathrm{EAC}$ is understudied.

Several genomic studies have investigated the genetic susceptibility of BE/EAC [5, 30-33]. Whole-exome sequencing studies have investigated the genomic alterations in a larger sample size and reported mutations in ELMO1 and DOCK2 [5, 34]. BE/EAC has persistently displayed heterogeneous clinical outcomes with an underlying genetic heterogeneity. In the study reported here, we attempt to merge multiple types of genomic changes, including repeats, TOHs, and insertions/deletions, across platforms to better understand the progression of BE to EAC.

\section{Results}

\section{Prediction of common TOH regions}

The common $\mathrm{TOH}(\mathrm{cTOH})$ regions were identified as described by Orloff et al. [35]. After false-discovery rate (FDR) adjustment for the effects of sex and population stratification factors, 24 cTOH regions on 13 chromosomes were found to be significantly differentially represented between $\mathrm{BE} / \mathrm{EAC}$ cases and controls with $P<0.05$ (Table 1). There are $13 \mathrm{cTOH}$ regions that are over-represented in the BE/EAC cases, as compared to controls, with odds ratios (OR) 2.38-8.36 (adjusted- $P=$ 0.002-0.045). In addition, there are $11 \mathrm{cTOH}$ regions under-represented in BE/EAC cases, as compared to controls $\quad(\mathrm{OR}=0.15-0.48, \quad$ adjusted- $P=0.004-0.038)$. The largest region of $\mathrm{CTOH}$ is on chromosome 13 that covers four Mbp and harbors the most number of SNPs (Table 1). The smallest cTOH region was identified on chromosome 20, the smallest chromosome.

\section{Distribution of IRs in the $\mathrm{CTOH}$ regions}

The significant $24 \mathrm{cTOH}$ regions were used as a guide to screen for the presence of repeats, and more specifically IRs, using exome sequence data generated from eight $\mathrm{BE} / \mathrm{EAC}$ patients that served as a validation series. The search yielded 61,858 predicted IRs with an average size of $4606 \mathrm{bp}$ (ranging from 39 to $21,947 \mathrm{bp}$ ). The most abundant of the predicted IRs were in the cTOH regions, located on chromosomes two and nine, while the least abundant IRs were located in the $\mathrm{cTOH}$ regions on chromosome 13 (Table 2). We found that the predicted IRs were disparately distributed within significant $\mathrm{cTOH}$ regions.

\section{Distribution of insertion/deletion in cTOH regions and across all samples}

The $\mathrm{TOH}$ regions harbor other types of genomic variants in addition to SNPs and repeat elements. Therefore, we sought to identify insertions and deletions by using the exome sequence data generated from the eight $\mathrm{BE} /$ EAC patients. We identified insertions and deletions within the $\mathrm{CTOH}$ regions, and their distributions varied in the eight BE/EAC patients (Fig. 1). We located 180 positions of insertions and deletions on genes across all cTOH regions. The lengths of the insertions and deletions for all samples ranged from 1 to $191 \mathrm{bp}$. One of the eight samples had a very high frequency of insertions and deletions. Overall, chromosomes 7 and 15 seemed to have longer insertions and deletions in $50 \%$ of the samples. However, in one of the samples, the longest 
Table 1 All predicted $\mathrm{CTOH}$ regions

\begin{tabular}{|c|c|c|c|c|c|}
\hline Chromosome regions & Length (bp) & No. of SNPs & $\begin{array}{l}\text { Adjusted } \\
P \text {-value }\end{array}$ & $\begin{array}{l}\text { Adjusted } \\
\text { FDR assoc. }\end{array}$ & Adjusted OR (95\%Cl) \\
\hline $1 \mathrm{p} 22.3$ & 381,139 & 113 & 0.025 & 0.653 & $4.65(1.22,17.81)$ \\
\hline 2p23.3 - p23.2 & $1,363,418$ & 165 & 0.021 & 0.888 & $0.32(0.12,0.84)$ \\
\hline 3p24.3 & $1,039,742$ & 238 & 0.015 & 0.315 & $2.78(1.22,6.32)$ \\
\hline 3p24.3 & 434,675 & 122 & 0.01 & 0.315 & $8.36(1.66,42.09)$ \\
\hline 3p24.1 & 628,410 & 100 & 0.023 & 0.315 & $0.21(0.06,0.81)$ \\
\hline $3 q 13.32$ & $1,263,994$ & 227 & 0.028 & 0.315 & $2.67(1.11,6.41)$ \\
\hline $4 q 12$ & 434,403 & 115 & 0.034 & 0.625 & $3.89(1.11,13.66)$ \\
\hline $4 q 13.1$ & 879,986 & 150 & 0.026 & 0.625 & $0.48(0.25,0.91)$ \\
\hline $6 p 12.3$ & $1,416,850$ & 181 & 0.03 & 0.554 & $0.39(0.17,0.92)$ \\
\hline $6 q 14.1$ & 384,341 & 102 & 0.039 & 0.554 & $5.3(1.09,25.82)$ \\
\hline $7 p 22.2$ & 632,597 & 187 & 0.045 & 0.707 & $2.84(1.03,7.85)$ \\
\hline 7p12.2 & 462,813 & 129 & 0.031 & 0.707 & $0.34(0.13,0.9)$ \\
\hline $8 q 21.11-q 21.12$ & $1,727,694$ & 238 & 0.023 & 0.653 & $2.72(1.15,6.43)$ \\
\hline $8 q 22.2$ & $1,589,641$ & 117 & 0.029 & 0.653 & $0.25(0.07,0.87)$ \\
\hline $9 q 21.13$ & $1,308,361$ & 255 & 0.036 & 0.333 & $0.46(0.22,0.95)$ \\
\hline $9 q 21.31$ & 491,323 & 107 & 0.026 & 0.333 & $0.25(0.08,0.85)$ \\
\hline $9 q 33.1$ & 958,487 & 265 & 0.013 & 0.333 & $2.48(1.21,5.08)$ \\
\hline $9 q 34.13$ & 367,627 & 107 & 0.037 & 0.333 & $0.18(0.04,0.9)$ \\
\hline $12 q 14.1$ & $1,242,069$ & 168 & 0.038 & 0.611 & $0.37(0.15,0.95)$ \\
\hline $13 q 21.1$ & $4,413,655$ & 608 & 0.002 & 0.05 & $2.38(1.36,4.16)$ \\
\hline $14 q 13.3-q 21.1$ & 866,302 & 149 & 0.012 & 0.252 & $3.1(1.28,7.49)$ \\
\hline $15 q 22.33-q 23$ & $1,142,927$ & 150 & 0.023 & 0.368 & $4.15(1.22,14.12)$ \\
\hline $18 q 12.1$ & 584,602 & 124 & 0.004 & 0.052 & $0.15(0.04,0.54)$ \\
\hline 20p12.1 & 267,272 & 104 & 0.029 & 0.29 & $4.22(1.16,15.39)$ \\
\hline
\end{tabular}

Table 2 Distribution of IRs and significant genes across $\mathrm{CTOH}$ regions

\begin{tabular}{|c|c|c|c|c|c|c|}
\hline Chromosome & No. of IRs & Min (bp) & $\operatorname{Max}(b p)$ & Average (bp) & No. of genes with IRs & Significant genes $^{\mathrm{a}}$ \\
\hline chr1 & 1362 & 39 & 14,668 & 4731 & 10 & MCOLN2, WDR63 ${ }^{b}$ \\
\hline chr2 & 11,340 & 41 & 11,425 & 5025 & 19 & \\
\hline chr3 & 5591 & 39 & 15,570 & 4512 & 18 & EOMES, $K A T 2 B^{b}, R^{b} B S 3^{b}$ \\
\hline chr4 & 6635 & 39 & 18,320 & 4451 & 12 & TMEM165 \\
\hline chr6 & 4166 & 39 & 17,214 & 4220 & 11 & \\
\hline chr7 & 4219 & 43 & 11,301 & 4728 & 24 & AMZ1, GNA12 ${ }^{b}$, IQCE, SDK1, SNX8, TTYH3 \\
\hline chr8 & 4440 & 39 & 21,947 & 4492 & 12 & C8orf84, HNF4G, VPS13B \\
\hline chr9 & 10,387 & 39 & 18,110 & 4711 & 33 & C9orf98, LAMC3, NUP214, RFK, RPSAP9, TMEM2, TLE1 \\
\hline chr12 & 1754 & 41 & 20,219 & 4064 & 6 & METTL1, MON2 ${ }^{b}$, USP15 \\
\hline chr13 & 1008 & 39 & 13,255 & 3672 & 0 & \\
\hline chr14 & 2186 & 39 & 19,438 & 4482 & 9 & CTAGE5 $^{b}$, SEC23A, SLC25A21 \\
\hline chr15 & 3852 & 39 & 11,243 & 4718 & 14 & $A A G A B, I Q C H, L R R C 49$ \\
\hline chr18 & 2711 & 39 & 13,381 & 4319 & 4 & B4GALT6 \\
\hline chr20 & 2207 & 39 & 12,552 & 4448 & 8 & C20orf7 \\
\hline
\end{tabular}

apresent in at least three out of eight samples

${ }^{b}$ Of the 33 genes, the pathway analysis prioritized eight genes to the NOTCH, transcription, inflammation, and signaling pathways of BE/EAC 


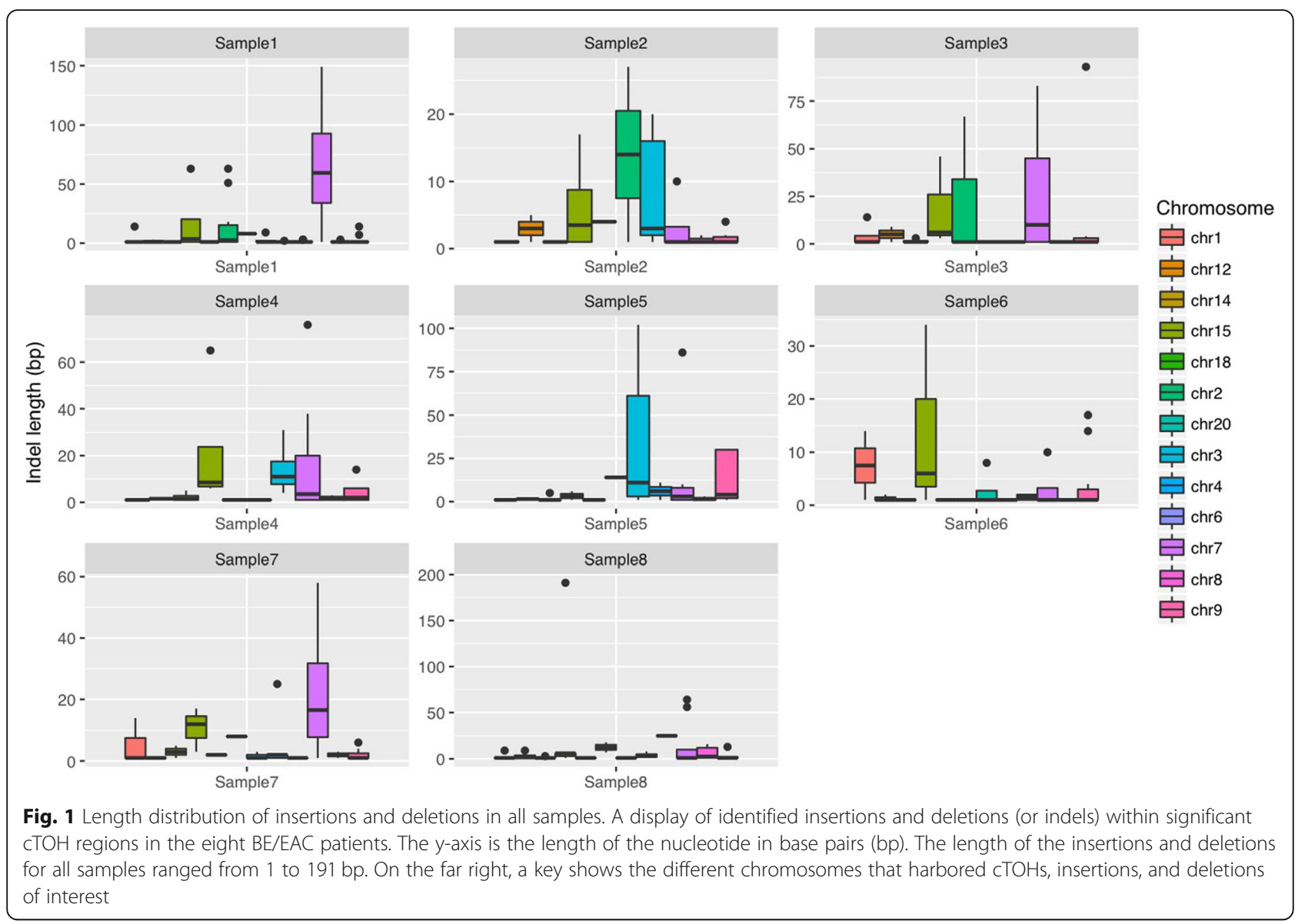

insertions and deletions were on chromosome 15 (Fig. 1). Chromosome 3 also had relatively long insertions and deletions in $38 \%$ of the samples. Shorter insertions and deletions were more frequent than longer insertions (Table 2).

\section{Mapping genes in significant $\mathrm{cTOH}$ regions that align with identified insertions/deletions and repeats using exome sequences}

The identified insertions and deletions were mapped on to the genomic regions containing the 33 genes, as displayed on the karyogram in Fig. 2. The genes that consistently showed insertions and deletions that overlapped across all samples were WDR63 (a WD repeat in domain 63), VPS13B (a vacuolar protein sorting homolog $13 \mathrm{~B}$ ), MON2 (a regulator to endosome-to-Golgi trafficking), and CTAGE5 (a cutaneous T-cell lymphoma-associated antigen 1), Additional file 1. Interestingly, we identified miR4423, located around 600 base pairs downstream of WDR63, which has previously been associated with airway epithelial cell differentiation and other cancers, e.g., lung cancer [36].
Further characterization of the short-listed genes and their roles in $\mathrm{BE} / \mathrm{EAC}$

The genes identified within the BE/EAC-related $\mathrm{cTOH}$ regions, including those that overlapped with the insertion/deletion and IRs, may have roles in either the development or progression of BE to EAC. MetaCore ${ }^{\text {TM }}$ bioinformatics software was used to analyze biological pathways as well as disease and gene networks that are associated with BE/EAC. Analysis of the short-listed genes revealed the top ten enriched pathways and networks (Figs. 3a and b). The top two pathways are the $\mathrm{NOTCH}$ signaling pathway and G (or guanine nucleotide-binding) protein-coupled signaling (Fig. 3a), followed by the endoplasmic reticulum (ER)-to-Golgi and WNT pathways (Fig. 3A). Transcriptional regulation and cholecystokinin signaling are the two top networks identified for BE/EAC (Fig. 3b), followed by $\mathrm{NOTCH}$ signaling, $\mathrm{ER}$, and inflammation protein $\mathrm{C}$ signaling, which are also important in BE/EAC (Fig. 3b).

The use of multiple data sources can help provide comprehensive information about the functional roles of the identified genes. Therefore, in addition to MetaCore, we also used the Comparative Toxicogenomics databases to analyze all the genes and miR-4423, which yielded 


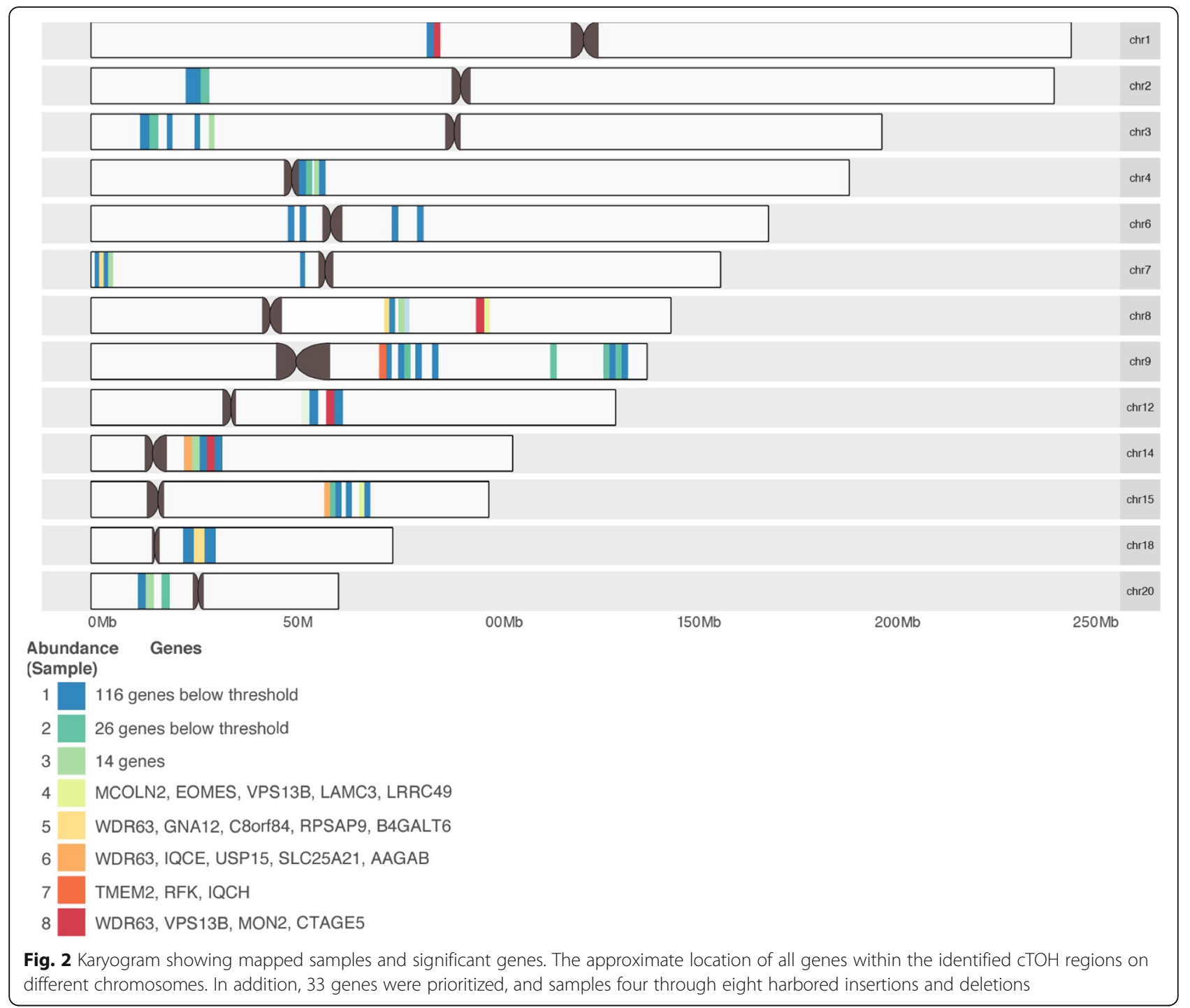

both complementary and supplementary results on key functional roles that affect $\mathrm{BE}$ and $\mathrm{EAC}$, as shown in Figs. 4a and b. Some of the overlapping pathways were $\mathrm{NOTCH}, \mathrm{WNT}$, and G-protein signaling pathways. The analysis also revealed differentially affected pathways in $\mathrm{BE}$ and EAC.

As with the MetaCore database, NOTCH and inflammation were persistently important. Differentially affected pathways were associated with the identified miR-4423 and were overrepresented in transcriptional regulation, $\mathrm{NOTCH}$ and cholecystokinin signaling, cell cycle regulation, and others (Figs. 4a and b). Overlap existed across $\mathrm{BE}, \mathrm{EAC}$, and miR-4423 pathways or processes.

By extracting functional information from multiple sources, we were able to verify and rank the importance of NOTCH signaling, WNT, inflammatory pathways, nuclear receptor signaling, nuclear degranulation, and cancer pathways to $\mathrm{BE}$ and EAC. Out of the 33 genes, 28 were involved in the cancer pathways and processes. The genes that were particularly important in these pathways were WDR63, GNA12, KAT2B, RBMS3, VPS13B, TLE1, MON2, and CTAGE5. In addition, miR-4423 seemed to have a key role among the identified pathways.

We then performed network analysis to identify interactions amongst the 33 genes relevant to $\mathrm{BE} / \mathrm{EAC}$, and found that 5 co-expressed genes out of 33 genes (WDR63, GNA12, RFK, B4GALT6, and LAMC3) were indeed part of the network (Fig. 5). LAMC3 was involved in extracellular matrix (ECM) receptor interaction and regulation of focal adhesion, which plays an important role in the maintenance of tissue structure and tissue morphogenesis. The interactions between cells and the ECM can regulate cellular activities, such as migration, proliferation, and apoptosis. GNA12 (G protein subunit 


\begin{tabular}{|c|c|c|c|c|}
\hline \multicolumn{5}{|c|}{$-\log 10(p-v a l u e)$} \\
\hline \multirow{6}{*}{$\begin{array}{r}\text { Development Notch Signaling Pathway } \\
\text { G protein-coupled receptors signaling in lung cancer } \\
\text { Riboflavin metabolism } \\
\text { Cytoskeleton remodeling Substance P mediated membrane blebbling } \\
\text { DeltaF508-CFTR traffic / ER-to-Golgi in CF } \\
\text { wtCFTR traffic / ER-to-Golgi (normal) }\end{array}$} & & $7.354 \mathrm{e}-4$ & $4.192 \mathrm{e}-2$ & $2 / 43$ \\
\hline & & $2.281 \mathrm{e}-3$ & $6.502 \mathrm{e}-2$ & $2 / 76$ \\
\hline & & $1.332 \mathrm{e}-2$ & $7.012 \mathrm{e}-2$ & $1 / 14$ \\
\hline & & $1.521 \mathrm{e}-2$ & $7.012 \mathrm{e}-2$ & $1 / 16$ \\
\hline & & $1.615 \mathrm{e}-2$ & $7.012 \mathrm{e}-2$ & $1 / 17$ \\
\hline & & $1.615 \mathrm{e}-2$ & $7.012 \mathrm{e}-2$ & $1 / 17$ \\
\hline \multirow{5}{*}{$\begin{array}{r}\text { Development WNT signaling pathway. Part 1. Degrading of beta-catenin in the absence WNT signaling } \\
\text { Development S1P4 receptor signaling pathway } \\
\text { G-protein signaling M-RAS regulation pathway } \\
\text { Stem cells Trophectoderm differentiation }\end{array}$} & & $1.803 \mathrm{e}-2$ & $7.012 \mathrm{e}-2$ & $1 / 19$ \\
\hline & & $2.085 \mathrm{e}-2$ & $7.012 \mathrm{e}-2$ & $1 / 22$ \\
\hline & & $2.273 \mathrm{e}-2$ & $7.012 \mathrm{e}-2$ & $1 / 24$ \\
\hline & & $2.367 \mathrm{e}-2$ & $7.012 \mathrm{e}-2$ & $1 / 25$ \\
\hline & 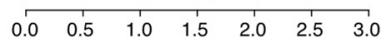 & & & \\
\hline Networks & $-\log 10(p-v a l u e)$ & $\mathrm{p}$-value & FDR & Ratio \\
\hline Transcription Nuclear receptors transcriptional regulation & & $1.022 \mathrm{e}-3$ & $2.452 \mathrm{e}-2$ & 3/195 \\
\hline Signal Transduction Cholecystokinin signaling & & $5.792 \mathrm{e}-3$ & $6.951 \mathrm{e}-3$ & 2/106 \\
\hline Signal transduction NOTCH signaling & & $2.661 \mathrm{e}-2$ & $2.129 \mathrm{e}-2$ & $2 / 235$ \\
\hline Protein folding ER and cytoplasm & & $4.838 \mathrm{e}-2$ & $2.513 \mathrm{e}-1$ & $1 / 44$ \\
\hline Signal Tranduction BMP and GDF signaling & & $9.778 \mathrm{e}-2$ & $2.513 \mathrm{e}-1$ & $1 / 91$ \\
\hline Inflammation Protein C signaling & & $1.151 \mathrm{e}-1$ & $2.513 \mathrm{e}-1$ & $1 / 108$ \\
\hline Cell adjesion integrin priming & & $1.171 \mathrm{e}-2$ & $2.513 \mathrm{e}-1$ & $1 / 110$ \\
\hline Cardiac development BMP TGF beta signaling & & $1.241 \mathrm{e}-1$ & $2.513 \mathrm{e}-1$ & $1 / 117$ \\
\hline Transcription Chromatin modification & & $1.331 \mathrm{e}-1$ & $2.513 \mathrm{e}-1$ & $1 / 126$ \\
\hline Signal transduction Androgen receptor nuclear signaling & & $1.331 \mathrm{e}-1$ & $2.513 \mathrm{e}-1$ & $1 / 126$ \\
\hline & $\begin{array}{llllll}0^{\prime} & 1.0 & 1.5 & 2.0 & 2.5 & 3.0\end{array}$ & & & \\
\hline \multicolumn{5}{|c|}{$\begin{array}{l}\text { Fig. } 3 \text { Display of pathways related to significant genes in CTOH regions. a Enriched pathways identified from EAC specific genes. Analysis of the } \\
\text { key prioritized genes revealed the top ten enriched pathways. The intensity of the blue color bars reflects the importance of the pathway in BE/ } \\
\text { EAC. The top two pathways are the NOTCH signaling pathway and G protein-coupled signaling pathway. Other important pathways include the } \\
\text { ER-to-Golgi and WNT pathways. b Enriched networks identified from EAC specific genes. Analysis of the key genes revealed the top ten } \\
\text { functionally enriched process networks. The intensity of the blue color bars reflects the importance of the network processes in BE/EAC. The top } \\
\text { nine network processes are likely important processes in BE/EAC. Transcriptional regulation and cholecystokinin signaling are the two top } \\
\text { network processes for BE/EAC. NOTCH signaling, ER, and inflammation protein C signaling processes seem to be important in BE/EAC }\end{array}$} \\
\hline
\end{tabular}

alpha 12) was found in the WNT signaling pathway. GNA12 can be upregulated by GPCR and then trigger RhoGEF, Rho, ROCK, and subsequently affect tissue invasion and metastasis. The second pathway was a metabolic pathway, where B4GALT6 and RFK were involved in glycan biosynthesis and metabolism.

\section{Discussion}

Genetic heterogeneity and the complex BE and EAC clinical outcomes have presented challenges in diagnosis and management of the $\mathrm{BE} / \mathrm{EAC}$. Whereas the importance of SNPs and their associations with disease risk are well established, clearly SNPs alone cannot completely unravel the complex link between the genome and the disease. More rigorous and inclusive genomic approaches are warranted to identify global contribution of the diverse genomic alterations in the development of $\mathrm{BE} / \mathrm{EAC}$. In this study, we use SNP data to screen for TOHs. Then, we integrated the exome sequence data within the $\mathrm{TOH}$ regions to identify IRs or direct repeats and insertion/deletions to prioritize genes and pathways that are important in BE/EAC. Our integrated analysis across platforms revealed genes that play a role in key significant pathways important to $\mathrm{BE}$ and $\mathrm{EAC}$ development and progression. These pathways were $\mathrm{NOTCH}, \mathrm{WNT}$, inflammatory pathways, nuclear receptor signaling, nuclear degranulation, and cancer pathways [21].

We observed that several genes from our list were associated with the development of $\mathrm{BE}$ or EAC and replicated previous studies. For example, the MetaCore pathway analysis linked GNA12 to inflammatory roles in $\mathrm{BE} / \mathrm{EAC}$. GNA12 has previously been shown to be upregulated in esophageal squamous cell carcinoma cells [37], which induces the carcinogenic effects of GNA12. Furthermore, GNA12 promotes tumor-cell invasion and metastasis by activating the RhoA/ROCK signaling pathway and upregulating proinflammatory cytokine production [38-41]. Interestingly, RBMS3, one of our candidate genes, has previously been shown to have a tumor-suppression function, through c-Myc downregulation, and contributed to poor prognosis in esophageal squamous cell carcinoma [42].

Emerging data now provide insights into the link among methylation of different repeat families, maintenance of chromosome structural integrity, and fidelity of normal transcriptional regulation [27]. Interestingly with the integrated data from IRs, insertion/deletions, and 


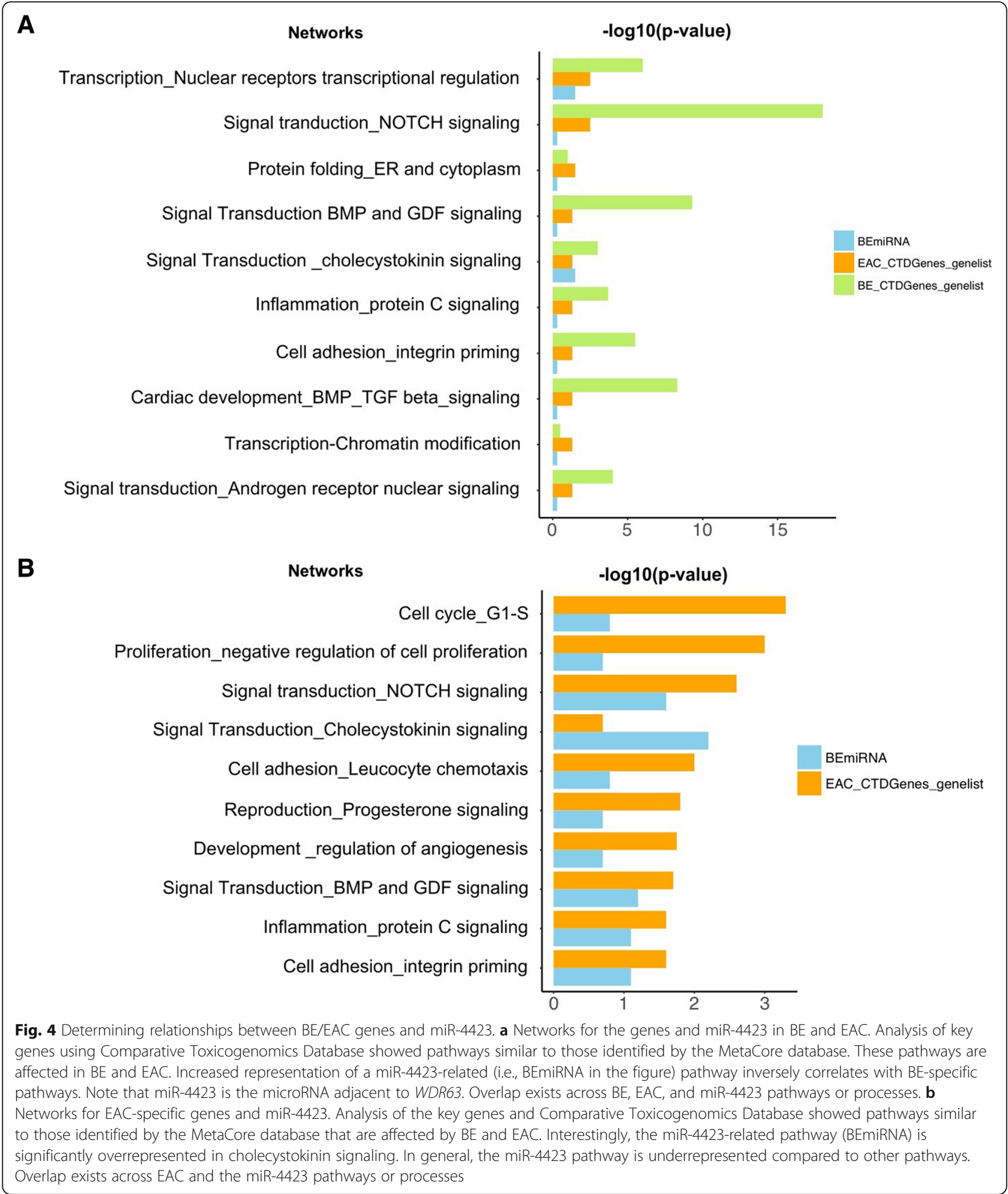

significant cTOHs, we were able to identify key genes that may have a role in BE/EAC. The frequencies of IRs, insertions, and deletions in the case-only exome data implies that the variants maybe important in $\mathrm{BE}$ and EAC. More specifically, WDR63, miR-4423, VPS13B,
MON2, and CTAGE5 consistently showed these overlapping variants. Previous studies have shown that hypomethylation is more prevalent in the repeat regions and has diverse ways of contributing to cancer behavior. For example, hypomethylation of repeated DNA sequences 


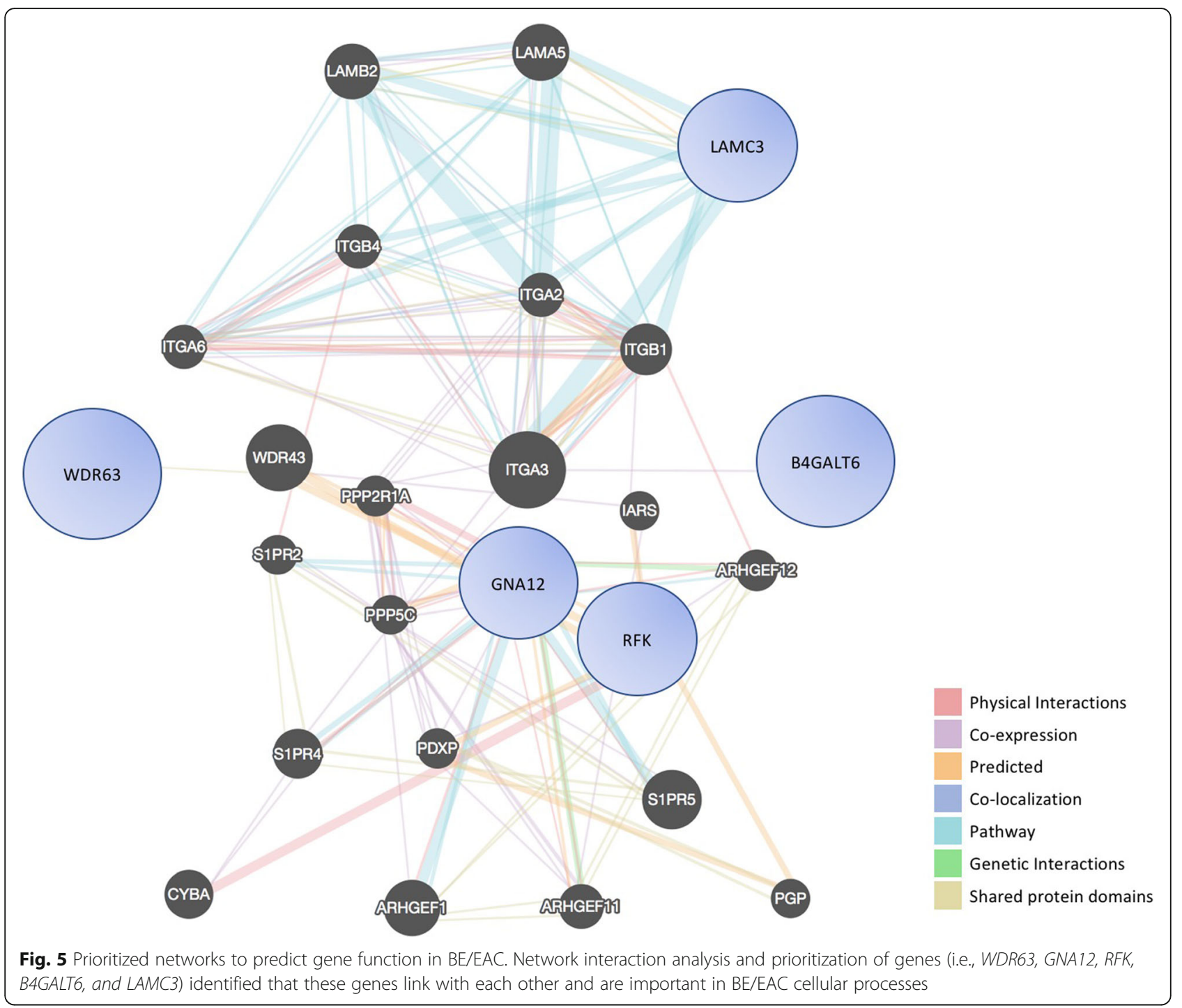

[43-46] is largely responsible for the global DNA hypomethylation that is frequently observed in cancers [4749]. Tandem centromeric satellite, centromere-adjacent satellite 2, the interspersed Alu, and long interspersed elements (LINE)-1 repeats are the most frequently studied DNA cancer hypomethylated repeats [44-52]. Further study is warranted to assess the nature of methylation patterns in the five key genes we identified and their correlation with the progression of $\mathrm{BE}$ to $\mathrm{EAC}$ or with the severity of the diseases. BE/EAC-associated aberrations in the miRNA and/or epigenetic patterns can explain the development and clinical stages of the diseases. Along the same lines, miR-4423 has been shown to regulate WDR63 and has previously been linked to airway epithelial cell differentiation and lung cancer [36].

Our pathway analysis showed similar results compared to previous multi-region whole-exome sequencing studies [53-55]. Chen et al. [53] reported similar pathways, such as the NOTCH signaling pathway and WNT pathway, when comparing a tumorous dysplasia cohort and a non-tumorous dysplasia cohort in mutational landscapes. The NOTCH signaling pathway has been associated with $C D X 2$ gene expression in the development of $\mathrm{BE}$ [56]. Our findings from the REACTOME database also indicate the importance of $\mathrm{NOTCH}$ signaling, based on our prioritized list of key genes. A previous publication showed that increased CDX2 expression [57] is driven by inhibiting $\mathrm{NOTCH}$ signaling during $\mathrm{BE}$ development [58]. Our MetaCore analysis also showed that HNF4G and TLEI are two genes that have a role in the $\mathrm{NOTCH}$ and WNT pathways and signal transduction. More importantly, TLEI and WDR63 have similar highly conserved C-terminal WD-repeat domains; hence, they will display similar functions. In addition to the independent role of pathways, crosstalk between WNT and $\mathrm{NOTCH}$ signaling plays an important role in cancer 
prognosis. The binding of secreted WNT ligands to the cysteine-rich domain of Frizzled (Fzd) family receptors stimulates the WNT signaling pathway [59], where we found the GNA12 gene (Fig. 4a).

For other carcinogenesis processes, the interplay of WDR63 and miR-4423 was reported to be associated with lung cancer [36, 60]. The mature forms of miR-4423 can co-express with WDR63 in mucociliary epithelium. WDR63 is downregulated in lung cancers, probably through DNA methylation. MiR-4423 regulates airway epithelium differentiation by repressing the Delta/Notch pathway [36]. Both miR-4423 and WDR63 can be affected by DNA damage or rearrangement (e.g., due to IRs) and stress-induced transcription factors. Our study is the first to report the possible carcinogenesis function of WDR63 and miR-4423 among BE/AC patients. Since lung cancer and esophageal cancer share similar risk factors, such as alcohol and tobacco use, and have similar histological subtypes, some genes may play similar roles in different types of cancer development.

\section{Conclusions}

This study highlights the importance of integrating $\mathrm{TOH}$ data with IRs to identify DNA rearrangements that can inform BE/EAC development. BE/EAC-specific microRNA expression, measured in readily collected samples, can be used for early BE/EAC detection. This data can be potentially integrated with other 'omics' data for a comprehensive understanding of complex susceptibility of BE/EAC.

\section{Methods}

\section{Selection of BE/ EAC patients}

This study was approved by the respective Institutional Review Boards for Research at each participating location where the research was performed. The study involved recruitment of all consenting adults with histological-proven $\mathrm{BE}$ and/or EAC as well as families with two or more cases with $\mathrm{BE}$ and/or EAC from both academic and community hospitals and clinics. Only white patients of Northern or Western European descent were selected and sex-matched between cases and controls.

\section{Genotyping and QC}

Germline genomic DNA samples obtained from white blood cells were genotyped using Human610-Quad BeadChips, after which the resulting genotypes were subjected to routine quality control steps: determination of missing genotype rate, testing for non-random genotyping failure, Hardy-Weinberg equilibrium, genotype call rates, MAF of $3-5 \%$, and finally checking for contamination from pipetting errors. Samples were screened and selected only if they had a minimum 95\% successful genotype call rate. SNPs with departures from Hardy-Weinberg equilibrium (HWE test, $P=0.0000001$ ), and missingness per SNP greater than 5\% were excluded from further analyses. As a result, 176 cases/192 controls (231 males/137 females) were kept. We used genotypes from Chr 1 22 only and in total, 570,044 SNPs genotypes were used.

\section{Assessment of population stratification}

Failure to account for population substructure may lead to both false positive and false negative SNP-disease associations [61]. BE/EAC has been reported to be highly prevalent in populations of European ancestry, but nonetheless, population stratification was analyzed, as previously described [5], by using the principal components analysis (PCA) module contained in EigenStrat $[62,63]$, and by using PLINK software [64]. Since the population was matched by race, we did not detect population substructure.

\section{Quantifying tracts of homozygosity and comparing frequencies in cancer cases and controls Identifying $\mathrm{TOH}$ and common $\mathrm{TOH}$ (cTOH) regions}

We used the method described in Orloff et al. [35]. The data from all research participants were examined to determine whether a minimum number of individuals shared a $\mathrm{TOH}$ call at a given position (Fig. 5). To identify statistical differences between TOHs within a case-control design, we only retained those TOHs in which 10 or more subjects shared 100 identical homozygous calls, which we operationally define as a common $\mathrm{TOH}(\mathrm{cTOH})$. A total of $644 \mathrm{cTOHs}$ were identified across the genome, ranging in size from 100 to 4827 SNPs in length (mean $=196, S D=221$, median $=147$, first quartile is 119 , and third quartile is 211 ), and from $136 \mathrm{~kb}$ to $15,410 \mathrm{~kb}$ (mean $=1160 \mathrm{~kb}, \mathrm{SD}=1445 \mathrm{~kb}$, median $=793 \mathrm{~kb}$, first quartile is $521 \mathrm{~kb}$, and third quartile is $1194 \mathrm{~kb})(16)$ to identify TOHs.

\section{Detection of $\mathrm{CTOH}$ that are associated with BE/EAC}

We then pursued testing for association between $\mathrm{CTOH}$ and $\mathrm{BE} / \mathrm{EAC}$. By considering each $\mathrm{CTOH}$ as a genomic variant, a genome-wide case-control analysis was conducted for each $\mathrm{cTOH}$, where a $\mathrm{cTOH}$ was viewed as a binary variable based on the presence or absence of a cTOH. A logistic model was fitted for each cTOH by considering disease status as the outcome and the cTOH as the predictor, and we adjusted for gender and population stratification factors. $P$-values were obtained by Wald tests and ORs (95\% CI) and were calculated through coefficient estimates of the fitted logistic model (Table 1).

\section{Analysis of BE/EAC exome and integration with $\mathrm{CTOH}$, insertions, deletions, and nucleotide repeats}

Whole-exome libraries from eight independent BE/EAC patients were prepared and sequenced. We followed the 
exome sequence pipeline from the Broad's Genome Analysis Tool Kit (GATK Version 3, best practices work flow) [65] to process the sequence data. Raw-exome sequence reads were mapped onto the human reference genome sequence build 36/hg18, downloaded from the University of California, Santa Cruz (UCSC) genome browser with the Burrows-Wheeler aligner [66] (BWA version v.0.6.1; http://bio-bwa.sourceforge.net).

Since the TOH regions likely harbor other types of genomic variants, we sought to identify insertions and deletions using the exome sequence data. Insertion or deletion (indel) realignment, base- and quality-score recalibrations from the resultant binary alignment map (BAM) files were performed with GATK, Sequence Alignment/Map [66] (SAMtools), and Picard. Variant discovery and indel calling were performed with the GATK Haplotype Caller. The high-quality sequences were assembled with the de novo assembler SPAdes, version 3.12.0, and compared with MEGAHIT.

For insertions or deletions to be significant, they had to appear in at least three out of the eight individuals who were sequenced, and they had to overlap with regions carrying inverted or simple repeats. Nucleotide repeat elements are abundant in the human genome and may have significant roles in disease development [4749]. Therefore, the reference genome was checked for the presence of simple repeats, using RepeatMasker 4.0.7, and IRs, using Inverted Repeats Finder (IRF) version 3.05 [67], to locate and/or predict locations of IRs in the exomic and/or flanking regions of genes located in the $\mathrm{TOH}$ regions. The minimap2 was then used to map assembled contigs from eight BE/EAC patient samples that served as a validation series on the reference genome and within $\mathrm{CTOH}$ regions.

Bedtools 2.27.0 was used to extract the overlapping regions from all data: reference genes, $\mathrm{cTOH}$ regions, nucleotide repeat elements, and contigs from all samples. In-house python scripts were written to automate the analysis pipeline from assembling exome data to mapping repetitive elements to identifying cTOH blocks and insertions/deletions for all eight germline samples (Fig. 6). We inspected all resultant variants through the

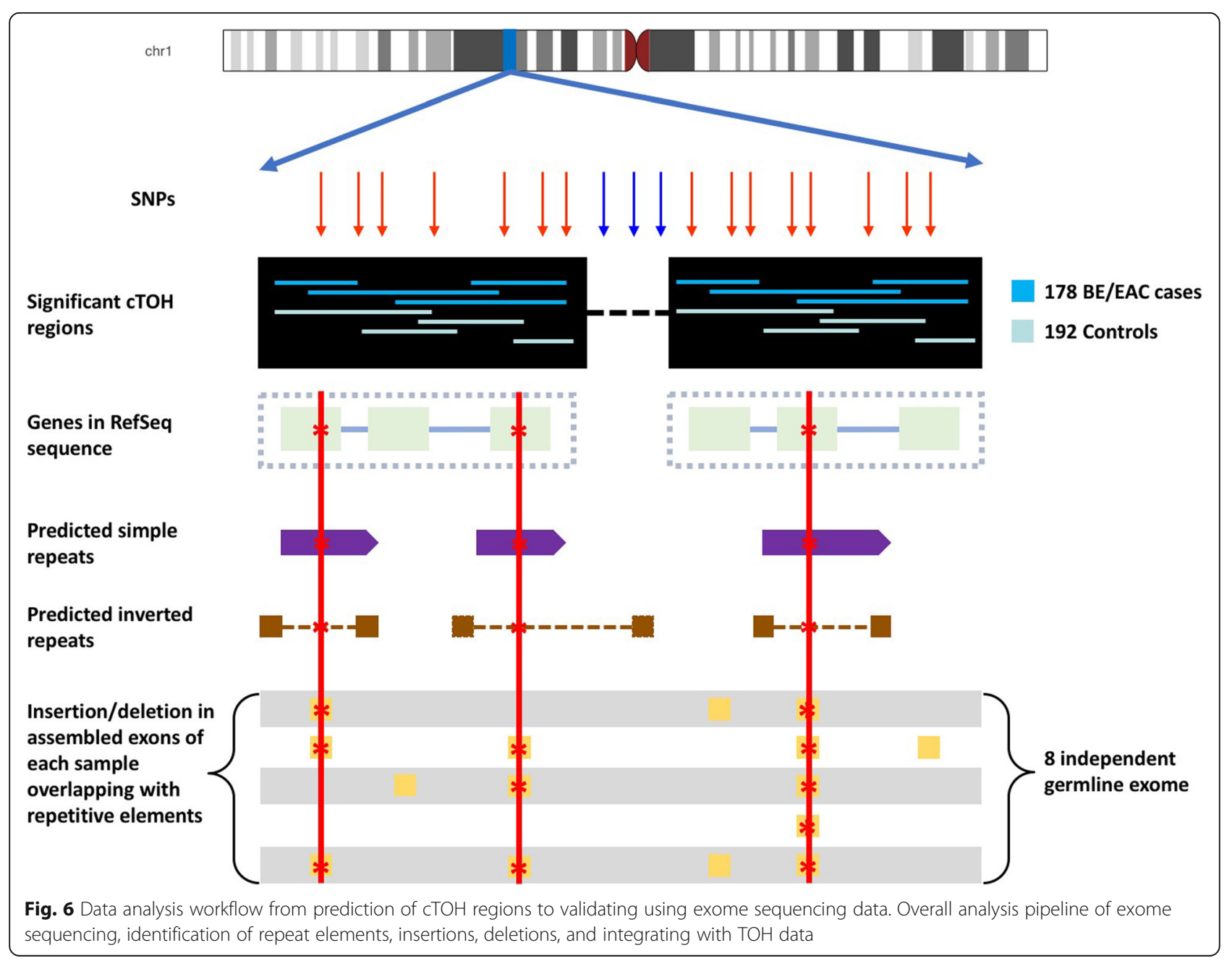


Integrative Genomics Viewer [68] (IGV; https://software.broadinstitute.org/software/igv/). The genes associated with $\mathrm{BE} / \mathrm{EAC}$ and containing insertion/deletion within contigs across all samples were collected in a tab-separated value (TSV) file and visualized using $R$ packages: ggplot2 and ggbio.

\section{Pathway and network analyses to predict functional roles in $\mathrm{BE} / \mathrm{EAC}$}

Since the key genes identified within $\mathrm{CTOH}$ regions that overlapped with the insertions/deletions and IRs may have possible roles in either the development or progression of $\mathrm{BE}$ to EAC, we used MetaCore bioinformatics software and curated Comparative Toxicogenomics Database to analyze biological pathways as well as disease and gene networks that are associated with BE/EAC. MetaCore contains an integrated pathway and network analysis for multi-omics types of data and also has a comprehensive systems biology analysis suite that helps identify high-quality experimental molecular interactions and pathways, gene disease associations, as well as chemical metabolism and toxicity information.

Network analysis was done using an open source GeneMANIA package, which builds and uses weighted gene interaction networks from various sources of data [69]. It uses a fast heuristic algorithm, derived from ridge regression, to integrate multiple functional association networks and predict gene function from a single process-specific network using label propagation. Genes that were significant from our $\mathrm{TOH}$ and exome analyses were analyzed to predict possible roles in BE and/or EAC.

\section{Additional file}

Additional file 1: Locations for genes within $\mathrm{CTOH}$ regions that also harbor indels. (XLSX $14 \mathrm{~kb}$ )

\section{Abbreviations}

BE: Barrett's esophagus; $\mathrm{CTOH}$ : Common Tracts of homozygosity; EAC: Esophageal cancer; GATK: Genome Analysis Tool Kit; TOH: Tracts of homozygosity

\section{Acknowledgements}

We would like to thank Tianjiao Shen for her contributions in this research.

\section{Funding}

Publication of this article was supported by funding from the Winthrop P. Rockefeller Cancer Institute at the University of Arkansas for Medical Sciences. The data used was secondary and research was not supported by any grant.

\section{Availability of data and materials}

All data generated or analyzed during this study are included in this published article and its supplementary information (i.e. Additional files 1). Additional data will be available upon request and has been submitted to the SRA database.

\section{About this supplement}

This article has been published as part of BMC Bioinformatics Volume 20 Supplement 2, 2019: Proceedings of the 15th Annual MCBIOS Conference. The full contents of the supplement are available online at https:// bmcbioinformatics.biomedcentral.com/articles/supplements/volume-20supplement-2.

\section{Authors' contributions}

WW performed the analysis and drafted the manuscript. JJ conducted the pathway analysis and discussed the results and interpretation of final data. EB analyzed the gene-gene interaction and discussed the results and interpretation of final data. MO conceived and performed analysis, directed the project, and drafted the manuscript. CE provided intellectual input, writing, and samples. All authors participated in finalizing and approving the manuscript.

\section{Ethics approval and consent to participate}

This study was approved by the Institutional Review Board for Research at each participating institution. The study involved national recruitment from academic and community hospitals and clinics of consenting adults with histological-proven $\mathrm{BE}$ and/or EAC as well as families with two or more cases with BE and/or EAC.

Consent for publication

Not applicable.

\section{Competing interests}

The authors declare that they have no competing interests.

\section{Publisher's Note}

Springer Nature remains neutral with regard to jurisdictional claims in published maps and institutional affiliations.

\section{Author details}

${ }^{1}$ Arkansas Center for Genomic Epidemiology \& Medicine and The Department of Biomedical Informatics, University of Arkansas for Medical Sciences, Little Rock, AR 72205, USA. ${ }^{2}$ The Department of Epidemiology, University of Arkansas for Medical Sciences, Little Rock, AR 72205, USA.

${ }^{3}$ Winthrop P. Rockefeller Cancer Institute, University of Arkansas for Medical Sciences, Little Rock, AR 72205, USA. ${ }^{4}$ Genomic Medicine Institute, Cleveland Clinic, Cleveland, OH 44195, USA.

Published: 14 March 2019

\section{References}

1. Garud SS, Keilin S, Cai Q, Willingham FF. Diagnosis and management of Barrett's esophagus for the endoscopist. Ther Adv Gastroenterol. 2010;3(4): 227-38.

2. Key Statistics for Esophageal Cancer [https://www.cancer.org/cancer/ esophagus-cancer/about/key-statistics.htm/\#references], $(8,2018)$.

3. Zhang J, Bowers J, Liu L, Wei S, Gowda GN, Hammoud Z, Raftery D. Esophageal cancer metabolite biomarkers detected by LC-MS and NMR methods. PLoS One. 2012;7(1):e30181.

4. Sharma N, Ho KY. Risk factors for Barrett's oesophagus. Gastrointestinal tumors. 2016:3(2):103-8.

5. Orloff M, Peterson C, He X, Ganapathi S, Heald B, Yang Y-r, Bebek G, Romigh $\mathrm{T}$, Song $\mathrm{JH}, \mathrm{Wu}$ W. Germline mutations in MSR1, ASCC1, and CTHRC1 in patients with Barrett esophagus and esophageal adenocarcinoma. JAmA. 2011;306(4):410-9.

6. Zheng H, Wang Y, Tang C, Jones L, Ye H, Zhang G, Cao W, Li J, Liu L, Liu Z. TP53, PIK3CA, FBXW7 and KRAS mutations in esophageal cancer identified by targeted sequencing. Cancer Genomics-Proteomics. 2016;13(3):231-8.

7. Chak A, Ochs-Balcom H, Falk G, Grady WM, Kinnard M, Willis JE, Elston R, Eng C. Familiality in Barrett's esophagus, adenocarcinoma of the esophagus, and adenocarcinoma of the gastroesophageal junction. Cancer Epidemiology and Prevention. Biomarkers. 2006;15(9):1668-73.

8. Gharahkhani P, Fitzgerald RC, Vaughan TL, Palles C, Gockel I, Tomlinson I, Buas MF, May A, Gerges C, Anders M. Genome-wide association studies in oesophageal adenocarcinoma and Barrett's oesophagus: a large-scale metaanalysis. The Lancet Oncology. 2016;17(10):1363-73.

9. Dumbovic G, Forcales S-V, Perucho M. Emerging roles of macrosatellite repeats in genome organization and disease development. Epigenetics. 2017; 12(7):515-26.

10. RepeatMasker Open-4.0 [http://www.repeatmasker.org], $(4,2018)$.

11. Gandin I, Faletra F, Faletra F, Carella M, Pecile V, Ferrero GB, Biamino E, Palumbo P, Palumbo O, Bosco P. Excess of runs of homozygosity is associated with severe cognitive impairment in intellectual disability. Genetics in Medicine. 2014;17(5):396.

12. Gibson J, Morton NE, Collins A. Extended tracts of homozygosity in outbred human populations. Hum Mol Genet. 2006;15(5):789-95. 
13. Kirin M, McQuillan R, Franklin CS, Campbell H, McKeigue PM, Wilson JF. Genomic runs of homozygosity record population history and consanguinity. PLoS One. 2010;5(11):e13996.

14. Mezzavilla M, Vozzi D, Badii R, Alkowari MK, Abdulhadi K, Girotto G, Gasparini P. Increased rate of deleterious variants in long runs of homozygosity of an inbred population from Qatar. Hum Hered. 2015;79(1):14-9.

15. Pippucci T, Magi A, Gialluisi A, Romeo G. Detection of runs of homozygosity from whole exome sequencing data: state of the art and perspectives for clinical, population and epidemiological studies. Hum Hered. 2014;77(1-4):63-72.

16. Feuk L, Carson AR, Scherer SW. Structural variation in the human genome. Nat Rev Genet. 2006;7(2):85.

17. Speed D, Cai N, Johnson MR, Nejentsev S, Balding DJ, Consortium U. Reevaluation of SNP heritability in complex human traits. Nat Genet. 2017;49(7):986.

18. Hannan AJ. Tandem repeats mediating genetic plasticity in health and disease. Nat Rev Genet. 2018:19(5):286.

19. Bacolod MD, Schemmann GS, Wang S, Shattock R, Giardina SF, Zeng Z, Shia J, Stengel RF, Gerry N, Hoh J. The signatures of autozygosity among patients with colorectal cancer. Cancer Res. 2008;68(8):2610-21.

20. Lencz T, Lambert C, DeRosse P, Burdick KE, Morgan TV, Kane JM, Kucherlapati R, Malhotra AK. Runs of homozygosity reveal highly penetrant recessive loci in schizophrenia. Proc Natl Acad Sci. 2007;104(50):19942-7.

21. Lu S, Wang G, Bacolla A, Zhao J, Spitser S, Vasquez KM. Short inverted repeats are hotspots for genetic instability: relevance to cancer genomes. Cell Rep. 2015;10(10):1674-80.

22. Treangen TJ, Salzberg SL. Repetitive DNA and next-generation sequencing: computational challenges and solutions. Nat Rev Genet. 2012;13(1):36.

23. Ting DT, Lipson D, Paul S, Brannigan BW, Akhavanfard S, Coffman EJ, Contino G, Deshpande V, lafrate AJ, Letovsky S. Aberrant overexpression of satellite repeats in pancreatic and other epithelial cancers. Science. 2011;331(6017):593-6.

24. Lavi B, Levy Karin E, Pupko T, Hazkani-Covo E. The prevalence and evolutionary conservation of inverted repeats in proteobacteria. Genome biology and evolution. 2018;10(3):918-27.

25. Achaz G, Coissac E, Netter P, Rocha EP. Associations between inverted repeats and the structural evolution of bacterial genomes. Genetics. 2003;164(4):1279-89.

26. Jurka J, Kapitonov W, Pavlicek A, Klonowski P, Kohany O, Walichiewicz J. Repbase update, a database of eukaryotic repetitive elements. Cytogenetic and genome research. 2005;110(1-4):462-7.

27. Ross JP, Rand KN, Molloy PL. Hypomethylation of repeated DNA sequences in cancer. Epigenomics. 2010;2(2):245-69.

28. Wooster R, Cleton-Jansen A-M, Collins N, Mangion J, Cornelis R, Cooper C, Gusterson B, Ponder B, Von Deimling A, Wiestler O. Instability of short tandem repeats (microsatellites) in human cancers. Nat Genet. 1994;6(2):152.

29. Achaz G, Coissac E, Viari A, Netter P. Analysis of intrachromosomal duplications in yeast Saccharomyces cerevisiae: a possible model for their origin. Mol Biol Evol. 2000;17(8):1268-75.

30. Blum A, Venkitachalam S, Guo Y, Kieber-Emmons AM, Ravi L, Chandar AK, Iyer PG, Canto MI, Wang JS, Shaheen NJ. RNA sequencing identifies transcriptionally-viable gene fusions in esophageal adenocarcinomas. Cancer research 2016:canres. 2016:0979.

31. Singh A, Chak A. Advances in the management of Barrett's esophagus and early esophageal adenocarcinoma. Gastroenterology report. 2015;3(4):303-15.

32. Sun X, Chandar AK, Canto MI, Thota PN, Brock M, Shaheen NJ, Beer DG, Wang JS, Falk GW, lyer PG. Genomic regions associated with susceptibility to Barrett's esophagus and esophageal adenocarcinoma in African Americans: the cross BETRNet admixture study. PLoS One. 2017;12(10):e0184962.

33. Sun X, Chandar AK, Elston R, Chak A. What we know and what we need to know about familial gastroesophageal reflux disease and Barrett's esophagus. Clin Gastroenterol Hepatol. 2014;12(10):1664-6.

34. Dulak AM, Stojanov P, Peng S, Lawrence MS, Fox C, Stewart C, Bandla S, Imamura Y, Schumacher SE, Shefler E. Exome and whole-genome sequencing of esophageal adenocarcinoma identifies recurrent driver events and mutational complexity. Nat Genet. 2013;45(5):478.

35. Orloff MS, Zhang L, Bebek G, Eng C. Integrative genomic analysis reveals extended germline homozygosity with lung cancer risk in the PLCO cohort. PLoS One. 2012;7(2):e31975.

36. Perdomo C, Campbell JD, Gerrein J, Tellez CS, Garrison CB, Walser TC, Drizik E, Si H, Gower AC, Vick J. MicroRNA 4423 is a primate-specific regulator of airway epithelial cell differentiation and lung carcinogenesis. Proc Natl Acad Sci. 2013;110(47):18946-51.

37. Ling ZQ, Mukaisho Kl, Yamamoto $\mathrm{H}$, Chen KH, Asano $\mathrm{S}$, Araki $Y$, Sugihara $\mathrm{H}$, Mao WM, Hattori T. Initiation of malignancy by duodenal contents reflux and the role of ezrin in developing esophageal squamous cell carcinoma. Cancer Sci. 2010;101(3):624-30.

38. Jian SL, Hsieh HY, Liao CT, Yen TC, Nien SW, Cheng AJ, Juang JL. Galpha(1, 2) drives invasion of oral squamous cell carcinoma through up-regulation of proinflammatory cytokines. PLoS One. 2013;8(6):e66133.

39. Kelly P, Moeller BJ, Juneja J, Booden MA, Der CJ, Daaka Y, Dewhirst MW, Fields TA, Casey PJ. The G12 family of heterotrimeric G proteins promotes breast cancer invasion and metastasis. Proc Natl Acad Sci U S A. 2006;103(21):8173-8.

40. Kelly P, Stemmle LN, Madden JF, Fields TA, Daaka Y, Casey PJ. A role for the G12 family of heterotrimeric $\mathrm{G}$ proteins in prostate cancer invasion. J Biol Chem. 2006:281(36):26483-90.

41. Yuan B, Cui J, Wang W, Deng K. Galpha12/13 signaling promotes cervical cancer invasion through the RhoA/ROCK-JNK signaling axis. Biochem Biophys Res Commun. 2016:473(4):1240-6.

42. Li Y, Chen L, Nie CJ, Zeng TT, Liu H, Mao X, Qin Y, Zhu YH, Fu L, Guan XY. Downregulation of RBMS3 is associated with poor prognosis in esophageal squamous cell carcinoma. Cancer research 2011:canres. 2010:4291.

43. Ehrlich M. DNA methylation in cancer: too much, but also too little. Oncogene. 2002;21(35):5400.

44. Ehrlich M, Woods CB, Yu MC, Dubeau L, Yang F, Campan M, Weisenberger DJ, Long T, Youn B, Fiala ES. Quantitative analysis of associations between DNA hypermethylation, hypomethylation, and DNMT RNA levels in ovarian tumors. Oncogene. 2006;25(18):2636.

45. Hoffmann MJ, Schulz WA. Causes and consequences of DNA hypomethylation in human cancer. Biochem Cell Biol. 2005;83(3):296-321.

46. Weisenberger DJ, Campan M, Long TI, Kim M, Woods C, Fiala E, Ehrlich M, Laird PW. Analysis of repetitive element DNA methylation by MethyLight. Nucleic Acids Res. 2005:33(21):6823-36.

47. Narayan A, Ji W, Zhang XY, Marrogi A, Graff JR, Baylin SB, Ehrlich M. Hypomethylation of pericentromeric DNA in breast adenocarcinomas. Int J Cancer. 1998;77(6):833-8.

48. G-z Q, Dubeau L, Narayan A, Mimi CY, Ehrlich M. Satellite DNA hypomethylation vs. overall genomic hypomethylation in ovarian epithelial tumors of different malignant potential. Mutation Research/Fundamental and Molecular Mechanisms of Mutagenesis. 1999;423(1):91-101.

49. G-z Q, Grundy PE, Narayan A, Ehrlich M. Frequent hypomethylation in Wilms tumors of pericentromeric DNA in chromosomes 1 and 16. Cancer Genet Cytogenet. 1999;109(1):34-9.

50. Florl A, Steinhoff C, Müller M, Seifert H, Hader C, Engers R, Ackermann R, Schulz W. Coordinate hypermethylation at specific genes in prostate carcinoma precedes LINE-1 hypomethylation. Br J Cancer. 2004;91(5):985.

51. Kim M-J, White-Cross JA, Shen L, JPJ I, Rashid A. Hypomethylation of long interspersed nuclear element-1 in hepatocellular carcinomas. Mod Pathol. 2009:22(3):442.

52. Rodriguez J, Vives L, Jorda M, Morales C, Munoz M, Vendrell E, Peinado MA. Genome-wide tracking of unmethylated DNA Alu repeats in normal and cancer cells. Nucleic Acids Res. 2007;36(3):770-84.

53. Chen X-X, Zhong Q, Liu Y, Yan S-M, Chen Z-H, Jin S-Z, Xia T-L, Li R-Y, Zhou A-J, Su Z. Genomic comparison of esophageal squamous cell carcinoma and its precursor lesions by multi-region whole-exome sequencing. Nat Commun. 2017:8(1):524.

54. Reya T. Regulation of hematopoietic stem cell self-renewal. Recent Prog Horm Res. 2003;58:283-96.

55. Shahi P, Seethammagari MR, Valdez JM, Xin L, Spencer DM. Wnt and Notch pathways have interrelated opposing roles on prostate progenitor cell proliferation and differentiation. Stem Cells. 2011;29(4):678-88.

56. Tamagawa $Y$, Ishimura N, Uno G, Yuki T, Kazumori H, Ishihara S, Amano $Y$, Kinoshita Y. Notch signaling pathway and Cdx2 expression in the development of Barrett's esophagus. Lab Investig. 2012;92(6):896.

57. Harada H, Nakagawa H, Oyama K, Takaoka M, Andl CD, Jacobmeier B, von Werder A, Enders GH, Opitz OG, Rustgi AK. Telomerase induces immortalization of human esophageal keratinocytes without p16INK4a Inactivation1 $1 \mathrm{NIH}$ grants R01-DK5337 (AKR), P01-DE12467 (AKR), P01-CA098101 (AKR), Deutsche Krebshilfe 10-1656-Op 1 and D/96/17197 (OGO), NIH R21 DK64249-01 (HN), AGAJFDHN Fiterman award and American Cancer Society (GHE), and NIH/ NIDDK Center for molecular studies in digestive and liver diseases (P30 DK50306). Mol Cancer Res. 2003;1(10):729-38.

58. Vega ME, Giroux V, Natsuizaka M, Liu M, Klein-Szanto AJ, Stairs DB, Nakagawa H, Wang KK, Wang TC, Lynch JP. Inhibition of Notch signaling enhances transdifferentiation of the esophageal squamous epithelium towards a Barrett's-like metaplasia via KLF4. Cell Cycle. 2014;13(24):3857-66. 
59. Moghbeli M, Abbaszadegan MR, Golmakani E, Forghanifard MM. Correlation of Wnt and NOTCH pathways in esophageal squamous cell carcinoma. Journal of cell communication and signaling. 2016;10(2):129-35.

60. Robles Al, Harris CC. A primate-specific microRNA enters the lung cancer landscape. Proc Natl Acad Sci. 2013;110(47):18748-9.

61. Knowler WC, Williams R, Pettitt D, Steinberg AG. Gm3; 5, 13, 14 and type 2 diabetes mellitus: an association in American Indians with genetic admixture. Am J Hum Genet. 1988;43(4):520.

62. Price AL, Patterson NJ, Plenge RM, Weinblatt ME, Shadick NA, Reich D. Principal components analysis corrects for stratification in genome-wide association studies. Nat Genet. 2006;38(8):904.

63. Reich D, Price AL, Patterson N. Principal component analysis of genetic data. Nat Genet. 2008:40(5):491.

64. Purcell S, Neale B, Todd-Brown K, Thomas L, Ferreira MA, Bender D, Maller J, Sklar P, De Bakker PI, Daly MJ. PLINK: a tool set for whole-genome association and population-based linkage analyses. Am J Hum Genet. 2007; 81(3):559-75.

65. McKenna A, Hanna M, Banks E, Sivachenko A, Cibulskis K, Kernytsky A, Garimella K, Altshuler D, Gabriel S, Daly M, et al. The genome analysis toolkit: a MapReduce framework for analyzing next-generation DNA sequencing data. Genome Res. 2010;20(9):1297-303.

66. Li H, Durbin R. Fast and accurate short read alignment with burrowswheeler transform. Bioinformatics. 2009;25(14):1754-60.

67. Warburton PE, Giordano J, Cheung F, Gelfand Y, Benson G. Inverted repeat structure of the human genome: the $X$-chromosome contains a preponderance of large, highly homologous inverted repeats that contain testes genes. Genome Res. 2004;14(10a):1861-9.

68. Thorvaldsdottir H, Robinson JT, Mesirov JP. Integrative genomics viewer (IGV): high-performance genomics data visualization and exploration. Brief Bioinform. 2013;14(2):178-92

69. Mostafavi S, Ray D, Warde-Farley D, Grouios C, Morris Q. GeneMANIA: a realtime multiple association network integration algorithm for predicting gene function. Genome Biol. 2008;9(Suppl 1):S4.

Ready to submit your research? Choose BMC and benefit from:

- fast, convenient online submission

- thorough peer review by experienced researchers in your field

- rapid publication on acceptance

- support for research data, including large and complex data types

- gold Open Access which fosters wider collaboration and increased citations

- maximum visibility for your research: over $100 \mathrm{M}$ website views per year

At $\mathrm{BMC}$, research is always in progress.

Learn more biomedcentral.com/submissions 